\title{
Wavelet-Based Evolutionary Response of Multispan Structures Including Wave-Passage and Site-Response Effects
}

\author{
Van-Nguyen Dinh"; Biswajit Basu ${ }^{2}$; and Ronald B. J. Brinkgreve ${ }^{3}$
}

\begin{abstract}
Stochastic seismic wavelet-based evolutionary response of multispan structures including wave-passage and site-response effects is formulated in this paper. A procedure to estimate site-compatible parameters of surface-to-bedrock frequency response function (FRF) by using finite-element analysis of the supporting soil medium is proposed. The earthquake energy content is represented by a composite power spectrum density function contributed by the surface-to-bedrock FRF and bedrock power spectra. A long span multisupport structure is subjected to spatially varying differential support motions where the spatial-variability is represented by bedrock parametric coherency models and time-lags. In addition to the time-lags from wave-passage effects, the site-response effects from different soil conditions at the supports are characterized by frequency-dependent time-lags. In an illustrative case study, a three-span, two-dimensional hangar frame is analyzed using the proposed formulations. The time-lags resulting from site-response effects and computed by different FRFs show different variation in trends and frequency content. The site-response effect is found to introduce additional frequency nonstationarity and leads to an increase in the frame responses but with slower attenuation in time. DOI: 10.1061/(ASCE)EM.1943-7889.0000708. @ 2014 American Society of Civil Engineers.
\end{abstract}

Author keywords: Seismic analysis; Amplitude and frequency nonstationary spatial variability; Site-response effect; Wavelets; Parametric frequency response function.

\section{Introduction}

The ground motions at a site are random processes attributed to the complex characteristics of the sources and paths of seismic waves. Moreover, they are apparently nonstationary in both amplitude and frequency from frequency-proportional velocities of seismic waves and from traveling paths consisting of soil layers having different properties. The structural responses undergreund-motions are consequently random and temporally and spectrally nonstationary, and they should therefore be stochastically represented by time-varying statistical quantities such as the evolutionary power spectral density function (PSDF). When statistical quantities representing ground motions (input) are given for evaluating stochastic structural responses (output), the input-output relations are needed. The random vibration theory is applicable only if the input is time-invariant. For time-varying inputs, wavelet techniques are suitable tools because they can provide a joint time-frequency representation simultaneously. Wavelet techniques have been used to formulate the input-output

\footnotetext{
${ }^{1}$ Postdoetoral Research Fellow, Dept. of Civil, Structural and Environmental Engineering, School of Engineering, Trinity College Dublin, Dublin 2, Ireland. E-mail: nguyendv@tcd.ie; nguyendhhh@yahoo.com

${ }^{2}$ Professor, Dept. of Civil, Structural and Environmental Engineering, School of Engineering, Trinity College Dublin, Dublin 2, Ireland (corresponding author). E-mail: basub@tcd.ie

${ }^{3}$ Associate Professor, Faculty of Civil Engineering \& Geosciences, Delft Univ. of Technology, 2628 CN Delft, Netherlands; and Manager, PLAXIS bv, Computerlaan 14, 2628 XK Delft, Netherlands. E-mail: r.brinkgreve@ plaxis.nl

Note. This manuscript was submitted on August 4, 2012; approved on September 11, 2013; published online on September 13, 2013. Discussion period open until ars is separate discussions must be submitted for individual papers. This paper is part of the Journal of Engineering Mechanics, (C) ASCE, ISSN 0733-9399/040سחבח(12)/ $\$ 25.00$.
}

relations of single degree-of-freedom systems (Basu and Gupta 1998, 2000) and multiple degree-of-freedom systems (Basu and Gupta 1997; Tratskas and Spanos 2003) where the input spatialvariation was excluded and proportional damping was assumed.

Earthquake-induced ground motions are spatially varying because of four distinct phenomena: incoherence, wave-passage, attenuation, and site-response effects (der Kiureghian 1996). The spatial-variation of ground motions has pronounced effects on structures. Stochastic input-output relations of multisupport structures subjected to spatially varying ground motions have been formulated by using random vibrations (Hao 1994; Loh and Ku 1995) where incoherence and wave-passage effects are considered and the ground motions are only nonstationary in amplitude. Dumanogluid and Soyluk (2003) and Zhang et al. (2009) considered incoherence, wave-passage, and site-response effects in their ground-motion spatial-variability models and carried out stochastic analyses of long-span bridges whereas the frequency nonstationarity of excitations was neglected. The siteresponse effect was shown to contribute considerably to the maximum response amplitudes. However, the influence of site-response effect on the frequency content of the responses of the bridge could not be investigated.

A more general and realistic input-output relation has been proposed by Chakraborty and Basu (2008) using a wavelet-based framework in which the ground motions are nonstationary in both amplitude and frequency, the excitation spatial-variation from the wave-passage effect is considered, and the nonstationarity in both amplitude and frequency of the output are evaluated. That work is extended in this paper to include the site-compatible earthquake energy and siteresponse effect of supporting soil media beneath the supports.

In the literature, by including a term in the coherency phase, the site-response effect is considered in formulating complex coherency functions (der Kiureghian 1996) and in simulating spatially varying nonstationary ground-motion time histories (Zerva 2009; Konakli and der Kiureghian 2012). While this approach is suitable for ground-motion simulation, it faces a difficulty in spectral analysis of 
evolutionary responses. The direct use of a complex function is not feasible for the second-order moment of the stochastic responses because it is a real quantity. Instead, an alternative approach is formulated in this paper to separately represent the lagged coherency and the phase. The site-response effect is proposed to be characterized by frequency-dependent time-lags. The wavelet-based spatial-variation model for ground motion to evaluate stochastic response incorporates parametric lagged coherency, wave-passage, and site-response effects in this paper.

For simulating spatially varying nonstationary ground motions and analyzing structural responses, the earthquake energy content has been generally characterized by stationary two-sided KanaiTajimi PSDF (Hao et al. 1989; Chakraborty and Basu 2008) and Clough-Penzien PSDF (Hao 1994; Deodatis 1996; Dumanogluid and Soyluk 2003; Zhang et al. 2009) among others. The KanaiTajimi and Clough-Penzien_PSDFs are most commonly used in both parameterization and simulation of seismic ground motions (Zerva 2009). However, when using these spectra, the bedrock is assumed to be rigid and the parameters for the structure sites are not estimated from the specific geological profiles, but are empirically assumed. Because the dynamic properties of the soil medium vary horizontally from site to site, such description of PSDF is unable to represent the frequency and characteristic damping of the real soil conditions. The effect of soil layer thickness is also not accounted for, i.e., the natural period of a soil layer is proportional to its thickness (Kramer 1996). A thicker layer of soft soil may exhibit lower frequencies close to the dominant ones of the seismic waves and cause larger amplification of the propagating waves than a thinner layer would. To account for the thickness of soil deposit (i.e., the depth of the bedrock), the earthquake energy content at a site is represented in this paper by a composite PSDF contributed by the surface-to-bedrock frequency response function (FRF) and the bedrock power spectrum. A procedure to estimate site-compatible FRF parameters by finite-element analysis (FEA) of the soil profile under each individual support is proposed in this paper and applied in the considered case study. This procedure overcomes some of the existing limitations by accounting for (1) soil property horizontal variation, and (2) effects of soil layer thickness in the FEA.
Using the proposed representations of ground-motion spatial- 4 variation in "Representation of Parametric Coherency Model for Ground Motions" and earthquake energy in "Site-Compatible PSD of Ground Motions in Soil Medium on Elastic Bedrock," the waveletbased stochastic models of spatially varying ground motions (input) and seismic evolutionary responses of multispan structures (output) are formulated in "Wavelet-Based Modeling of Spatially Varying Ground Motions including Wave-Passage and Site-Response Effects" and "Wavelet-Based Evolutionary Responses of Multispan Structures Subjected to Differential Support Motions including Wave-Passage and Site-Response Effects," respectively (see also "Formulation for Calculation of Evolutionary Responses"). "Wavelet Basis Function" reviews an efficient wavelet basis function to be used in this paper. A flowchart explaining the proposed methodology and the relationship among the equations presented in this paper is provided in Fig. 1.

In the case study presented in "Numerical Example," a threespan, two-dimensional (2D) hangar frame supported on a horizontally varying property soil layer and a thick elastic bedrock layer is analyzed using the proposed formulations. The parameters of the Clough-Penzien and Kanai-Tajimi FRFs compatible to the site beneath each individual support are estimated. The stationary PSD at each support is calculated by using the parametric Kanai-Tajimi and Clough-Penzien FRFs. The stochastic processes corresponding to these PSDs are used as orthogonal processes at different supports for wavelet-based modeling of spatially varying ground motions and for wavelet-based evolutionary response analyses of the frame. The time-lags computed by using Kanai-Tajimi parametric FRFs vary by a moderate amount around a higher frequency whereas the time-lags computed by using Clough-Penzien parametric FRFs vary dramatically around a lower frequency possibly stemming from the additional lower frequency filter. Comparing with the results in the case when only wave-passage effect is considered, the site-response effect leads to an increase in wavelet-based root mean squares (RMS) of the frame relative displacements with a slower attenuation in time. The frequency content of such responses exhibits stronger nonstationary and their instantaneous PSD peaks are higher.

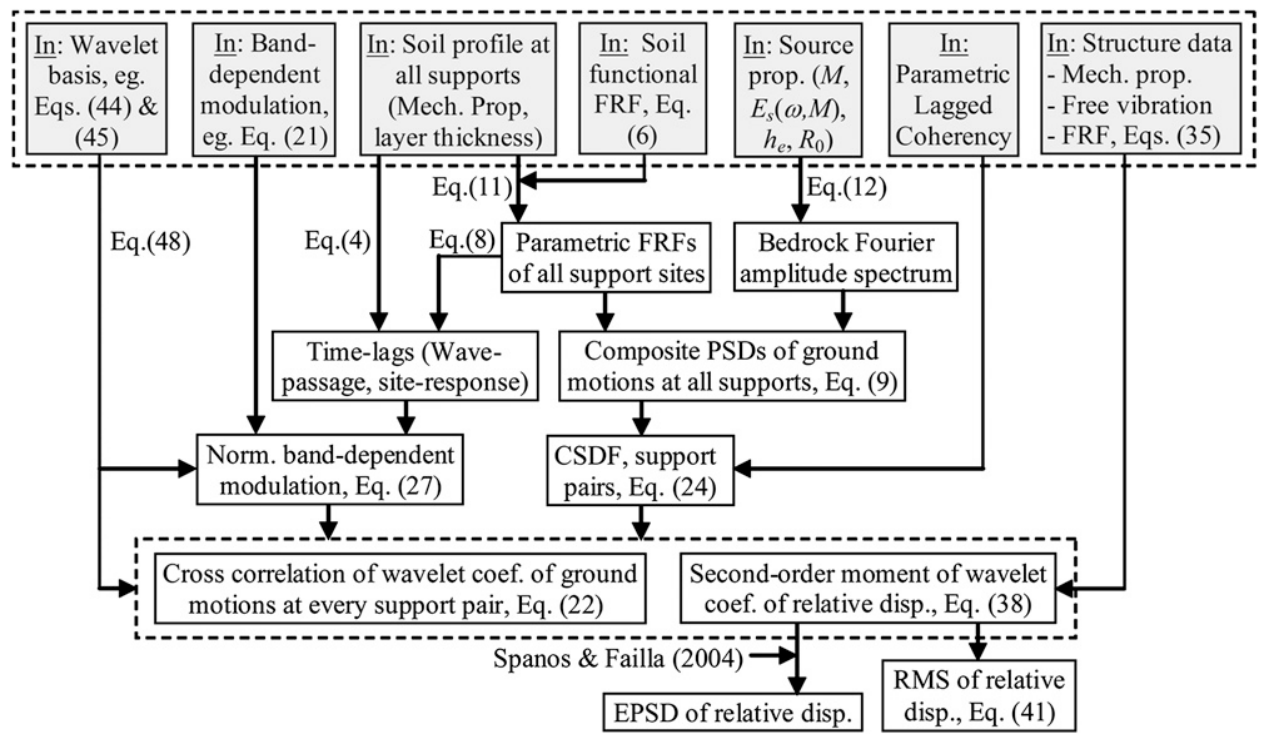

Fig. 1. Flowchart of the proposed methodology: coef. $=$ coefficients; CSDF $=$ cross-spectral density function; disp. $=$ displacement; FRF $=$ frequency response function; in. $=$ input; mech. $=$ mechanical; norm. $=$ normalized; prop. $=$ properties; $\mathrm{PSD}=$ power spectral density; $\mathrm{RMS}=$ root mean square 


\section{Representation of Parametric Coherency Model for Ground Motions}

The representation of ground-motion spatial variation by a proposed introduction of frequency-dependent time-lags for site-response effects and accounting for effects of incoherence and wave-passage is developed in this section.

The coherency function characterizes the spatial variability of ground-motions in a frequency domain. Considering two supports $r$ and $l$, the coherency function is represented by the cross-spectral density function $\hat{S}_{r l}(\omega)$ of the stationary parts of the ground motions at the two supports, normalized by the square-root of the corresponding PSDFs, i.e., $S_{r r}(\omega)$ and $S_{l l}(\omega)$, as (der Kiureghian 1996; Zerva and Zervas 2002)

$$
\gamma_{r l}(\omega)=\frac{\hat{S}_{r l}(\omega)}{\sqrt{S_{r r}(\omega) S_{l l}(\omega)}}
$$

The PSDF of ground motion at a support is related to the FRF of the soil layer beneath that support, and the bedrock PSDF in Eq. (9). Eq. (1a) can be written in a complex variable form as

$$
\gamma_{r l}(\omega)=\left|\gamma_{r l}(\omega)\right| \exp \left[i \theta_{r l}(\omega)\right]
$$

where the real term, $\left|\gamma_{r l}(\omega)\right|, 0 \leq\left|\gamma_{r l}(\omega)\right| \leq 1$, is the lagged coherency characterizing the variation in space. In the literature, $\left|\gamma_{r l}(\omega)\right|$ has been represented by common functions such as the ones given, for example, by Harichandran and Vanmarcke (1986), Luco and Wong (1986), and Hao et al. (1989) . In this paper, the parametric coherency models are estimated by using FE-based seismic analysis of a geological soil medium model including the bedrock. The coherency phase $\theta_{r l}(\omega)$ represents the difference in phase of the excitations at the two supports. When the wave-passage and siteresponse effects denoted by the superscripts $w p$ and site, respectively are considered, $\theta_{r l}(\omega)$ is expressed as

$$
\theta_{r l}(\omega)=\theta_{r l}^{w p}+\theta_{r l}^{\text {site }}(\omega)=-\omega t_{r l}^{w p}+\theta_{r l}^{\text {site }}(\omega)
$$

The use of representation in Eq. (1b) for spectral analysis of evolutionary excitations and structural responses faces the difficulty that the complex coherency cannot be directly introduced into any real-valued second-order moment quantity. To overcome this difficulty, the coherency phase $\theta_{r l}(\omega)$ is transformed into frequencydependent time-lags as

$$
\theta_{r l}(\omega)=-\omega . t_{r l}(\omega)=-\omega\left[t_{r l}^{w p}+t_{r l}^{\mathrm{site}}(\omega)\right]
$$

Consider a single soil layer under two surface sites $r$ and $l$ as shown in Fig. 2. The properties of the soil layer are horizontally varying. The propagation of seismic shear waves from the bottom to

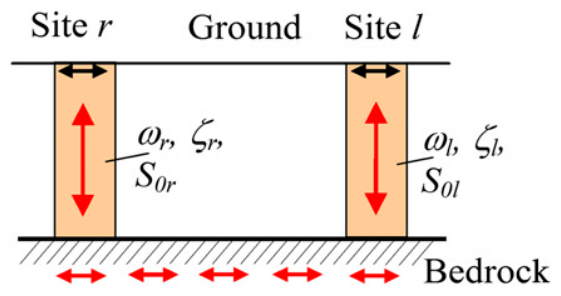

Fig. 2. Two-column model for site-response effects of a single layer of soil the top can be characterized by one-dimensional wave propagation. The time-lag from the wave-passage effect in Eqs. (2) and (3) is computed from the separation distance $\xi_{r l}$ and the wave-propagation velocities $V_{r}$ and $V_{l}$ beneath supports $r$ and $l$, respectively, and is given as

$$
t_{r l}^{w p}=\frac{2 \xi_{r l}}{V_{r}+V_{l}}
$$

The site-response effect between sites $r$ and $l$ is attributed to the difference in phases $\theta_{r}$ and $\theta_{l}$ at the two sites as (der Kiureghian 1996)

$$
\theta_{r l}^{\text {site }}(\omega)=\theta_{l}(\omega)-\theta_{r}(\omega)=\tan ^{-1} \frac{\operatorname{Im}\left[H_{l}(\omega) H_{r}^{*}(\omega)\right]}{\operatorname{Re}\left[H_{l}(\omega) H_{r}^{*}(\omega)\right]}
$$

When the behavior of the soil column is dominated by its first mode or when the high-frequency components of the ground motion do not have significant contribution to the structural responses, the functional form of the FRF at a site $H_{s}(\omega), s=r, l$ in Eq. (5), can be represented by the Kanai-Tajimi (K-T) filter function in Eq. (6) or the Clough-Penzien (C-P) filter function in Eq. (7) (Clough and Penzien 2003)

$$
\begin{gathered}
H_{s}^{K-T}(\omega)=\frac{1+2 i \varsigma_{s}\left(\omega / \omega_{s}\right)}{1-\left(\omega / \omega_{s}\right)^{2}+2 i \varsigma_{s}\left(\omega / \omega_{s}\right)} \\
H_{s}^{C-P}(\omega)=H_{s}^{K-T}(\omega) \frac{\left(\omega / \omega_{f}\right)}{1-\left(\omega / \omega_{f}\right)^{2}+2 i \varsigma_{f}\left(\omega / \omega_{f}\right)}
\end{gathered}
$$

In Eqs. (6) and (7), $\omega_{\mathrm{s}}=$ soil characteristic frequency; and $\zeta_{\mathrm{s}}$ $=$ damping ratio. The frequency $\omega_{f}$ and damping ratio $\zeta_{f}$ used in Clough-Penzien FRF, in Eq. (7), greatly attenuates the very low frequency components. The frequency-dependent time-lag from the site-response effect in Eq. (3) is given as

$$
t_{r l}^{\mathrm{site}}(\omega)=-\frac{1}{\omega} \theta_{r l}^{\mathrm{site}}(\omega)
$$

The maximum time-lags of ground motions at every support pair $r$ and $l$, from site-response effects, can be evaluated by substituting $\omega \approx\left(\omega_{s, r}+\omega_{s, l}\right) / 2$ for Kanai-Tajimi FRF or $\omega \approx\left(\omega_{f, r}+\omega_{f, l}\right) / 2$ for Clough-Penzien FRF into Eq. (8). The time-lags $t_{r l}^{w p}$ and $t_{r l}^{\text {site }}(\omega)$ contributing to the coherency phase $\theta_{r l}(\omega)$ will be used separately from the lagged coherency $\left|\gamma_{r l}(\omega)\right|$ in the following sections.

\section{Site-Compatible PSD of Ground Motions in a Soil Medium on Elastic Bedrock}

In this paper, the geological profile consists of a soil layer on a very thick elastic bedrock layer. The PSDF of ground motions at a support $r$ is related to that of bedrock motions by (der Kiureghian 1996)

$$
S_{r r}(\omega)=\left|H_{r}(\omega)\right|^{2} S^{\text {bedrock }}(\omega)
$$

where $H_{r}(\omega)=$ FRF of the soil layer beneath support $r$; and $S^{\text {bedrock }}(\omega)=$ PSD of bedrock. What follows later in the section is a proposed technique based on FE modeling of the soil medium to estimate the parameters of this FRF represented by a parametric form. The PSD of bedrock is expressed as 


$$
S^{\text {bedrock }}(\omega)=\frac{1}{T^{\text {bedrock }}}\left|F^{\text {bedrock }}(\omega)\right|^{2}
$$

where $T^{\text {bedrock }}=$ stationary duration of the bedrock excitation stochastic process contributed by the earthquake source and the source-to-bedrock path (Trifunac and Brady 1975). The estimation of soil FRF parameters and the formulation of Fourier amplitude spectrum of motions at the top level of the bedrock (hereafter in short called the bedrock), $F^{\text {bedrock }}(\omega)$, are presented in the following sections.

\section{Proposed Procedure to Estimate Site-Compatible FRF Parameters}

A procedure to estimate the FRF parameters of a single-layered soil column beneath a support $r$ by using FEA has been proposed. The soil column is modeled and analyzed using FEs. The software PLAXIS 2D (Brinkgreve et al. 2008) is used in this paper. The excitation is a white-noise acceleration uniformly applied at the bottom of soil layer. Assuming that the duration of the stationary motion at the bedrock level and at the surface level is the same, the unsmoothed absolute values of FRF of accelerations at the surface points with respect to the source (bottom) are computed from the Fourier amplitude ratio as

$$
\left|\tilde{H}_{r, i}\left(\omega_{k}\right)\right|=\frac{\left|F_{r, i}^{\text {surface }}\right|}{\left|F_{r, i}^{\text {bed }}\left(\omega_{k}\right)\right|} ; \quad k=1, \ldots, N_{\omega} ; i=1, \ldots, N_{\text {points }}
$$

where $N_{\omega}=$ number of discrete frequency intervals necessary around support $r$; and $N_{\text {points }}=$ number of surface points considered around support $r$. The value of $N_{\omega}$ should be chosen as a power of two to avoid zero-pad effects on fast-Fourier transform (Dinh and Basu 2012). The smoothed absolute values of FRF, $\left|\bar{H}_{r, i}\left(\omega_{k}\right)\right|$, $k=1, \ldots, N_{\omega}$, are obtained by averaging over $N_{\text {points }}$ the number of surface points considered around support $r$. The parameters of the FRF are estimated by fitting such smoothed absolute values to the functional form of the FRF, for example the Kanai-Tajimi spectrum in Eq. (6) or the Clough-Penzien spectrum in Eq. (7). The parametric fitting (nonlinear least square) in the software MATLAB is used for this purpose. Model parameters $\left(\omega_{\mathrm{s}}, \zeta_{\mathrm{s}}\right.$ for Kanai-Tajimi FRF or $\omega_{\mathrm{s}}$, $\zeta_{s}, \omega_{f}, \zeta_{f}$ for Clough-Penzien FRF) of the soil layer are obtained. After comparing the statistical parameters ( $R$-square, root-meansquare error (RMSE) $\underline{1}_{2}$ a final set of estimated model parameters and a parametric form $\left|H_{r}(\omega)\right|$ of a soil column model at support $r$ is obtained for each FRF functional form. This procedure is repeated for each individual support having different local soil conditions.

\section{Fourier Amplitude Spectrum of Earthquake Bedrock Motions}

The Fourier amplitude spectrum of earthquake motions at bedrock is represented by using the stochastic seismic spectrum (Boore 2003)

$$
\left|F^{\text {bedrock }}(\omega)\right|=C \cdot E_{S}(\omega, M) \cdot G(R) \cdot P(\omega, R) \cdot A(\omega) \cdot D(\omega)
$$

where the scaling factor and the source spectrum are, respectively, expressed as

$$
C=\frac{R_{e} V_{e} F_{s}}{4 \pi \rho_{0} V_{s 0}^{3}}
$$

$$
E_{s}(\omega, M)=\omega^{2} M_{0}\left\{\frac{1-\varepsilon}{1+\left[\omega / \omega_{a}(M)\right]^{2}}+\frac{\varepsilon}{1+\left[\omega / \omega_{b}(M)\right]^{2}}\right\}
$$

in which $R_{e}=$ radiation pattern; $V_{e}=$ partition of total shear wave energy into horizontal components; $F_{s}=$ constraint factor; $V_{\mathrm{s} 0}=$ shear wave velocity; $\rho_{0}=$ density of the source rock; $\omega_{a}$ $=$ lower-corner frequency of the source duration; $\omega_{b}=$ highercorner frequency at which the spectrum attains one-half of the highfrequency amplitude level; and $\varepsilon=$ weighting parameter. The moment magnitude $M$ is mapped from the seismic moment $M_{0} \mathbf{5}$ (dyn/em). The geometrical spreading function $G(R)$ is characterized by empirical formulas well supported by data of distance range from 10 to $1,000 \mathrm{~km}$ with

$$
R=\sqrt{R_{0}^{2}+h_{e}^{2}}
$$

where $R_{0}=$ epicentral distance; and $h_{e}=$ source depth.

For Eq. (12), $P(\omega, R)=$ path-dependent attenuation factor and is dependent on propagation velocity; $D(\omega)=$ diminution factor that accounts for the path-independent attenuation of high-frequency waveforms and can be represented by the $\kappa$-filter; and $A(\omega)$ $=$ amplification factor (which is approximated by the source/site impedance ratio in a numerical scheme using the quarter-wavelength approximation method).

\section{Wavelet-Based Modeling of Spatially Varying Ground Motions including Wave-Passage and Site-Response Effects}

A wavelet-based modeling of spatially varying ground motions including wave-passage and site-response effects has been proposed in this section. In wavelet analysis, a time series $u(t)$ is represented as a composition of several time-localized shifted and scaled wavelets (so-called the baby wavelets) $\psi_{a, b}(t)$ of a basic wavelet $\psi(t)$, where

$$
\psi_{a, b}(t)=\frac{1}{\sqrt{|a|}} \psi\left(\frac{t-b}{a}\right)
$$

The parameter $b$ localizes the basis function at $t=b$ and its neighborhood, and the parameter $a$ controls the frequency content of the basis function by stretching or compressing it. The discrete wavelet transform has been used to simulate ground motions (Iyama and Kuwamura 1999). Although the discrete wavelet transform is the most efficient and compact, its power-of-two relationship in scale fixes its frequency resolution (Gurley and Kareem 1999). Thus, the continuous wavelet transform (CWT), which allows more closely spaced scaling than the $2^{i}$ relationship, is used in this paper. The CWT convolves the signal $u(t)$ with a set of baby wavelets as (Basu and Gupta 1997, 1998, 2000)

$$
W_{\psi} u(a, b)=\frac{1}{\sqrt{|a|}} \int u(t) \psi^{*}\left(\frac{t-b}{a}\right) d t
$$

where the asterisk = complex conjugate. Eq. (15) gives the localized frequency information of $u(t)$ around $t=b$. The value $W_{\psi}(\cdot)$ maps a finite energy signal from the time domain to a finite energy $2 \mathrm{D}$ distribution in the scale-translation domain.

A set of differential nonstationary ground motions $\ddot{u}_{g r}(t)$, $r=1, \ldots, N_{s}$, at $N_{s}$ supports of a multispan structure is considered. In practice, $\ddot{u}_{g r}(t)$ is an evolutionary random process and can be expressed as (Priestley 1981) 


$$
\ddot{u}_{g r}(t, \omega)=\int_{-\infty}^{\infty} A^{r}(t, \omega) e^{i \omega t} d G_{r}(\omega)
$$

where $A^{r}(t, \omega)=$ slowly varying time- and frequency-dependent modulation; and $d G_{r}(\omega)=$ orthogonal increment process associated with the $r$ th support such that

$$
\begin{gathered}
E\left[d G_{r}(\omega) d G_{r}^{*}\left(\omega^{\prime}\right)\right]=0, \quad \omega \neq \omega^{\prime} \\
E\left[\left|d G_{r}(\omega)\right|^{2}\right]=S_{r r}(\omega) d \omega
\end{gathered}
$$

In Eq. (18), $S_{r r}(\omega)=$ two-sided PSDF of the stationary part of the random process, which has been formulated in Eq. (9). The evolutionary random process in Eq. (16) can be transformed in wavelet domain by using Eq. (16), and the wavelet coefficients at a discretized scale $a_{j}$ can be expressed as (Chakraborty and Basu 2008)

$$
W_{\psi} \ddot{u}_{g r}\left(a_{j}, b\right)=A_{j}^{r}(b) \int_{-\infty}^{\infty} e^{i \omega b} d \tilde{G}_{r}(\omega)
$$

where orthogonal increment process $d \tilde{G}_{r}(\omega)$ satisfies (Spanos and Failla 2004)

$$
\begin{gathered}
E\left[d \tilde{G}_{r}(\omega) d \tilde{G}_{r}^{*}\left(\omega^{\prime}\right)\right]=0, \quad \omega \neq \omega^{\prime} \\
E\left[\left|d \tilde{G}_{r}(\omega)\right|^{2}\right]=2 \pi a_{j}\left|\hat{\psi}\left(\omega a_{j}\right)\right|^{2} S_{r r}(\omega) d \omega
\end{gathered}
$$

The function $A_{j}^{r}(b)$ represents the amplitude modulation for $\ddot{u}_{g r}(t, \omega)$ at a scale $a_{j}$ that can be the extended Shinozuka-Sato amplitude modulation (Shinozuka and Sato 1967) given as

$$
A_{j}^{r}(b)=\alpha_{j}^{r}\left(e^{-\beta_{j}^{r} t}-e^{-\lambda_{j}^{r} t}\right)
$$

in which $\alpha_{j}^{r}, \beta_{j}^{r}$, and $\gamma_{j}^{r}=$ parameters of the amplitude modulation for the ground motion at the $j$ th band of frequency and at the $r$ th support.

Multiplying both sides of Eq. (19) by the complex conjugate corresponding to another support $l$, as being carried out by Chakraborty and Basu (2008) and considering the frequencydependent time-lags from site-response effects, the cross correlation of the wavelet coefficients of seismic ground motions at the two supports $r$ and $l$ and at a scale $a_{j}$ is

$$
\begin{aligned}
& E\left[W_{\psi} \ddot{u}_{g r}\left(a_{j}, b\right) W_{\psi} \ddot{u}_{g l}\left(a_{j}, b\right)\right] \\
& \quad=A_{j}^{r}(b) \int_{-\infty}^{\infty} A_{j}^{r}\left[b-t_{r l}^{w p}-t_{r l}^{\text {site }}(\omega)\right] E\left[d \tilde{G}_{r}(\omega) d \tilde{G}_{l}^{*}(\omega)\right]
\end{aligned}
$$

where $t_{r l}^{w p}=$ time-lag from the wave-passage effect; and $t_{r l}^{\text {site }}(\omega)$ $=$ frequency-dependent time-lag from site-response effects presented in Eqs. (4) and (8); and

$$
\begin{gathered}
E\left[d \tilde{G}_{r}(\omega) d \tilde{G}_{l}^{*}\left(\omega^{\prime}\right)\right]=0, \quad \omega \neq \omega^{\prime} \\
E\left[d \tilde{G}_{r}(\omega) d \tilde{G}_{l}^{*}(\omega)\right]=2 \pi a_{j}\left|\hat{\psi}\left(\omega a_{j}\right)\right|^{2} S_{r l}(\omega) d \omega
\end{gathered}
$$

The modulus of the cross-spectral density function between the ground motions at two supports $r$ and $l$ is written from Eq. (1a) as

$$
S_{r l}(\omega)=\left|\hat{S}_{r l}(\omega)\right|=\left|\gamma_{r l}(\omega)\right| \sqrt{S_{r r}(\omega) S_{l l}(\omega)}
$$

In this paper, the earthquake energy transmitted to each support $r$ is completely represented by its stationary PSDF $S_{r r}(\omega)$ in Eq. (9). Thus, the energy of the modulation must be unit-normalized before convoluting with the power spectral densities in Eq. (19). The energy content, $I_{A}$, of a frequency-dependent modulation before normalizing $A(t, \omega)$ is given by

$$
I_{A}=\int_{0}^{T} \int_{0}^{\infty}[A(t, \omega)]^{2} d \omega d t=\sum_{k=1}^{N_{\omega}} \sum_{n=1}^{N_{t}}\left[A\left(t_{n}, \omega_{k}\right)\right]^{2} \Delta \omega \cdot \Delta t
$$

The energy content is expressed in a band-dependent form as

$$
I_{A}=\sum_{j=1}^{m_{a}}\left\{N_{\omega}^{j} \sum_{n=1}^{N_{t}}\left[A_{j}\left(t_{n}\right)\right]^{2}\right\} \Delta \omega \cdot \Delta t
$$

where $m_{a}=$ number of frequency bands; $N_{\omega}^{j}=$ number of discrete frequencies in $j$ th band; and $N_{t}=$ number of time intervals. Hence, the unit-energy normalized amplitude modulation at a band $j$ is given by

$$
\bar{A}_{j}\left(t_{n}\right)=\frac{A_{j}\left(t_{n}\right)}{\sqrt{I_{A}}}
$$

\section{Wavelet-Based Evolutionary Responses of Multispan Structures Subjected to Differential Support Motions Including Wave-Passage and Site-Response Effects}

\section{Formulation for Calculation of Evolutionary Responses}

A formulation for calculating the evolutionary response including wave-passage and site-response effects is derived in this section. Consider a structure having $N$ degrees of freedom (DOFs) and $N_{s}$ supports subjected to spatially varying excitation-time histories $\ddot{u}_{g r}(t), r=1, \ldots, N_{s}$. The structure is modeled in a FE framework leading to a discrete dynamical system model. Using the consistent mass matrix approach and an assumption that the effect of the entire velocity-damping coupling is negligible in comparison with that of the inertia, the motion equations of the structure is given by (Clough and Penzien 2003)

$$
\mathbf{M} \ddot{\mathbf{u}}+\mathbf{C} \dot{\mathbf{u}}+\mathbf{K u}=-\left[\mathbf{M E}+\mathbf{M}_{g}\right] \ddot{\mathbf{u}}_{g}
$$

where $\mathbf{u}(t)=$ displacement vector relative to the support motions; $\mathbf{M}=$ system $N \times N$ mass matrix; $\mathbf{C}=N \times N$ damping matrix; and $\mathbf{K}=N \times N$ stiffness matrix. In Eq. (28), the $N \times N_{s}$ influence coefficient matrix $\mathbf{E}$, whose $k$ th column represents the displacements at the unconstrained DOF when a support DOF is displaced by a unit amount while all other support DOFs remain fixed, is expressed as $\mathbf{E}=-\mathbf{K}^{-1} \mathbf{K}_{g}$. The $N \times N_{s}$ matrices $\mathbf{M}_{g}$ and $\mathbf{K}_{g}$ account for the coupling of the inertia and stiffness between structural DOFs and ground motion DOFs and 


$$
\ddot{\mathbf{u}}_{g}=\left\langle\ddot{u}_{g_{1}}(t) \ldots \ddot{u}_{g_{N_{s}}}(t)\right\rangle^{T}
$$

$$
\ddot{z}_{k}+2 \eta_{k} \omega_{k} \dot{z}_{k}+\omega_{k}^{2} z_{k}=\sum_{r=1}^{N_{s}} \chi_{k}^{r} \ddot{u}_{g_{r}}(t), \quad k=1, \ldots, N
$$

In Eqs. (29) and (30), $z_{k}=k$ th modal component of the generalized coordinate vector $\mathbf{z}$; and the right-hand-side load $=$ sum of $k$ th modal earthquake load over all supports, with $\chi_{k}^{r}$ representing the $k$ th modal excitation factor at support $r$. In Eq. (29), $\gamma_{k}=$ complex modal stiffness for the nonproportional damping case. In Eq. (30), $\omega_{k}=$ modal frequency for the proportional damping case; and $\eta_{k}$ $=$ damping ratio for the proportional damping case.

Transforming Eqs. (29) and (30) by a chosen wavelet basis $\psi_{a, b}(t)$ and using Eq. (19) and the relations $\partial / \partial b\left[W_{\psi} z_{k}\left(a_{j}, b\right)\right]$ $=W_{\psi} \dot{z}_{k}\left(a_{j}, b\right)$ and $\partial^{2} / \partial b^{2}\left[W_{\psi} z_{k}\left(a_{j}, b\right)\right]=W_{\psi} \ddot{z}_{k}\left(a_{j}, b\right)$ gives

$$
\begin{aligned}
& \frac{\partial}{\partial b} W_{\psi} z_{k}\left(a_{j}, b\right)+\gamma_{k} W_{\psi} z_{k}\left(a_{j}, b\right)=\sum_{r=1}^{N_{s}} \chi_{k}^{r} A_{j}^{r}(b) \int_{-\infty}^{\infty} e^{i \omega b} d \tilde{G}_{r}(\omega) \\
& \frac{\partial^{2}}{\partial b^{2}} W_{\psi} z_{k}\left(a_{j}, b\right)+2 \eta_{k} \omega_{k} \frac{\partial}{\partial b} W_{\psi} z_{k}\left(a_{j}, b\right)+\omega_{k}^{2} W_{\psi} z_{k}\left(a_{j}, b\right) \\
& =\sum_{r=1}^{N_{s}} \chi_{k}^{r} A_{j}^{r}(b) \int_{-\infty}^{\infty} e^{i \omega b} d \tilde{G}_{r}(\omega)
\end{aligned}
$$

Solving Eqs. (31a) and (31b) (which resemble the equations of motion in wavelet domain) by using Duhamel's integral gives

$$
W_{\psi} z_{k}\left(a_{j}, b\right)=\sum_{r=1}^{N_{s}} \chi_{k}^{r} \int_{-\infty}^{+\infty} M_{r_{j}}^{k}(\omega, b) e^{i \omega b} d \tilde{G}_{r}(\omega)
$$

where

$$
M_{r_{j}}^{k}(\omega, b)=\int_{0}^{b} h_{k}(b-\tau) A_{j}^{r}(\tau) e^{i \omega(\tau-b)} d \tau
$$

where

$$
\bar{f}_{j}(\omega)=2 \pi a_{j} \bar{A}_{j}^{l}\left[b-t_{r l}^{w p}-t_{r l}^{s i t e}(\omega)\right] \cdot\left|H_{k}(\omega)\right|^{2} S_{r l}(\omega)\left|\hat{\psi}\left(a_{j} \omega\right)\right|^{2}
$$

The terms $\bar{A}_{j}^{r}(b)$ and $\bar{A}_{j}^{l}\left[b-t_{r l}^{w p}-t_{r l}^{\text {site }}(\omega)\right]=$ unit-energy normalized amplitude modulations for $\ddot{u}_{g_{r}}(t)$ and $\ddot{u}_{g_{l}}(t)$ at a scale $j$, respectively. By using the second-order moments obtained from Eq. (38), the EPSP of the relative displacement along $p$ th DOF can be estimated (Spanos and Failla 2004).

Based on the time-lags considered, for seismic waves already arrived at support $r$ but yet to arrive at support $l$, the modulation intensity at the latter support should be zero, i.e.

$$
\bar{A}_{j}^{l}\left[b-t_{r l}^{w p}-t_{r l}^{\text {site }}(\omega)\right]=0 \quad \text { if } b<t_{r l}^{w p}+t_{r l}^{\text {site }}(\omega)
$$

The instantaneous mean-square value of a time-dependent process (Basu and Gupta 1998) is used to compute that of the relative displacement along the $p$ th degree of freedom as

$$
\left.E\left[u_{p}^{2}(t)\right]\right|_{t=b_{i}}=K \sum_{j}^{m_{a}} \frac{E\left[W_{\psi}^{2} u_{p}\left(a_{j}, b_{i}\right)^{2}\right]}{a_{j}}
$$

where the term $K$ is expressed as

$$
K=\frac{\sigma^{2}-1}{4 \pi C_{\psi} \sigma}
$$

where $H_{k}(\omega)=$ conventional frequency-response function in the $k$ th mode, which is given for the nonproportional and proportional damping cases in the respective equations 


$$
C_{\psi}=\int_{-\infty}^{+\infty} \frac{|\hat{\psi}(\omega)|^{2}}{\omega} d \omega<\infty
$$

\section{Wavelet Basis Function}

Although in theory the proposed stochastic seismic evolutionary response is applicable for any wavelet basis function satisfying the admissibility criterion in Eq. (43), the choice of the basic function is important for the efficiency and accuracy in computation. Besides, the analysis resulted from CWT relies heavily on the scale discretizations and the selected frequency range (Kijewski-Correa and Kareem 2006). Several wavelet basis functions were shown advantageous in characterizing ground motions such as the Mexican hat wavelets (Zhou and Adeli 2003) and harmonic wavelets (Spanos et al. 2005). Tratskas and Spanos (2003) modeled the nonstationary base-excitations and estimate the stochastic evolutionary responses by using harmonic wavelets. Harmonic wavelets were also used by Spanos and Kougioumtzoglou (2012) to compute statistically linearized evolutionary responses of nonlinear oscillators subject to stochastic excitation. The modified Littlewood-Paley (MLP) basis function (Basu and Gupta 1998) is used in this paper because it provides high accuracy in spectral analysis (Spanos and Failla 2004) and advantages in numerical computation by enabling energy computation of any signal with nonoverlapping frequency bands. The MLP wavelet basis pair in time and frequency domain is given by

$$
\begin{array}{cl}
\psi(t)=\frac{1}{\pi \sqrt{2 F_{1}(\sigma-1)}} \cdot \frac{\sin \left(2 \pi F_{1} \sigma t\right)-\sin \left(2 \pi F_{1} t\right)}{t} \\
|\hat{\psi}(\omega)|=\frac{1}{\sqrt{4 \pi F_{1}(\sigma-1)}}, & F_{1} \leq\left|\frac{\omega}{2 \pi}\right| \leq \sigma F_{1} \\
=0 & \text { otherwise }
\end{array}
$$

where $F_{1}=$ initial cutoff frequency of the mother wavelet. If $F_{1}=0.5 \mathrm{~Hz}$, Eqs. (44) and (45) are reduced to the original forms of MLP basis function (Basu and Gupta 1998) as

$$
\begin{array}{rlrl}
\psi(t) & =\frac{1}{\pi \sqrt{\sigma-1}} \cdot \frac{\sin (\pi \sigma t)-\sin (\pi t)}{t} \\
|\hat{\psi}(\omega)| & =\frac{1}{\sqrt{2 \pi(\sigma-1)}}, & & \pi \leq|\omega| \leq \sigma \pi \\
& =0 & & \text { otherwise }
\end{array}
$$

The scaled Fourier transform is

$$
\begin{aligned}
\left|\hat{\psi}\left(\omega a_{j}\right)\right| & =\frac{1}{\sqrt{4 \pi F_{1}(\sigma-1)}} & & \text { when } \frac{2 \pi F_{1}}{a_{j}} \leq|\omega| \leq \frac{2 \pi F_{1} \sigma}{a_{j}} \\
& =0 & & \text { otherwise }
\end{aligned}
$$

The admissibility criterion coefficient, $C_{\psi}$, in Eq. (43) becomes

$$
C_{\psi}=\frac{1}{2(\sigma-1) \pi} \int_{-\infty}^{+\infty} \frac{1}{|\omega|} d \omega=\frac{\ln \sigma}{2(\sigma-1) \pi}
$$

It is noted by Basu and Gupta (1998) that for $\sigma=2^{1 / n}, n \geq 4$ is found reasonable, based on investigations on several ground motions recorded. However, because a small value of $\sigma$ leads to increased computational effort, a value of $\sigma=2^{1 / 4}$ has been chosen (Basu and Gupta 1998, 2000; Spanos and Failla 2004). A higher value of $\sigma$ can also be chosen in the case of ground motion with relatively smooth Fourier spectra.

\section{Numerical Example}

An application of the proposed theory and derived formulations in this paper relating to evolutionary response of structures with wavepassage and site-response effects is presented in this section. To illustrate the wave-passage and the site-response effects on the stochastic evolutionary responses, multispan structures that exhibit considerable vertical and horizontal responses should be examined. A three-span 2D frame of a hangar shown in Fig. 3 is therefore considered. The cross-sectional area and moment of inertia of the columns are $A_{c}=1.2 \mathrm{~m}^{2}$ and $I_{c}=0.144 \mathrm{~m}^{4}$, respectively; and those of the beams are $A_{b}=2.0 \mathrm{~m}^{2}$ and $I_{b}=0.667 \mathrm{~m}^{4}$, respectively. The frame material parameters are elastic modulus $E_{b}=2.0 \times 10^{11} \mathrm{~N} / \mathrm{m}^{2}$, Poisson ratio $\nu_{b}=0.29$, mass density $\rho_{b}=7,860 \mathrm{~kg} / \mathrm{m}^{3}$, and the modal damping ratios for the first two modes are $\zeta_{1}=\zeta_{2}=0.02$. The first five natural frequencies of the frame are $6.72,10.02,12.07$, 15.67 , and $33.96 \mathrm{rad} / \mathrm{s}$. A geological profile of the area beneath the supports is shown in Fig. 3 and the data is presented in Table 1. An earthquake is assumed to occur with the moment magnitude $M=5.5$, source depth $h_{e}=20 \mathrm{~km}$, and epicenter distance $R_{0}=100 \mathrm{~km}$ from the bridge.

Using the procedure presented in "Site-Compatible PSD of Ground Motions in a Soil Medium on Elastic Bedrock," four soil models representing the sites beneath four supports are analyzed by FEs using PLAXIS $2 D$ where each soil domain is modeled with dimensions $100 \times 50 \mathrm{~m}$. Fine meshes are used for discretization of the FE model with an automatic mesh generation scheme. Nine surface observation points $\left(N_{\text {points }}=9\right)$ located around the model centerline have been chosen. The model domain width is chosen as large as $100 \mathrm{~m}$ to reduce the effect of reflected waves at the vertical

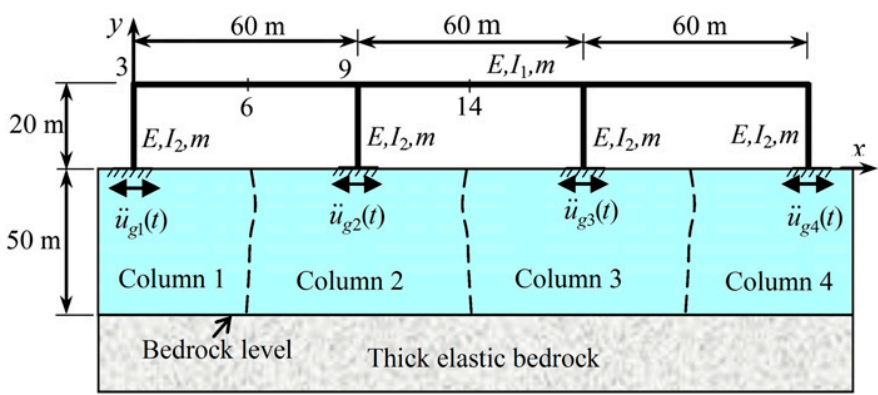

Fig. 3. Three-span frame subjected to spatially varying ground motions and geological profiles beneath the supports; 3, 6, 9, and 14 are the observation points

Table 1. Properties of Soil Layers, Bedrock, and Source Rock

\begin{tabular}{lcccr}
\hline Soil column & $E\left(\mathrm{MN} / \mathrm{m}^{2}\right)$ & $v$ & $\rho\left(\mathrm{kN} / \mathrm{m}^{3}\right)$ & $V_{s}(\mathrm{~m} / \mathrm{s})$ \\
\hline 1 & 24.6 & 0.25 & 17.9 & 73.42 \\
2 & 40.6 & 0.23 & 19.2 & 91.81 \\
3 & 21.6 & 0.22 & 18.2 & 68.89 \\
4 & 35.6 & 0.24 & 18.8 & 86.53 \\
Bedrock & 3,000 & 0.25 & 2,500 & 565.68 \\
Source rock & 70,533 & 0.23 & 2,800 & 3,200 \\
\hline
\end{tabular}

Note: $V_{s}=$ shear wave velocity; $\rho=$ density of the rock. 
boundaries on the observation points. A linear elastic soil model has been used for analysis. For generation of the FRFs, $N_{\omega}=1,024$ has been chosen. The estimated parameters of Clough-Penzien and Kanai-Tajimi FRFs compatible to the sites beneath the supports are shown in Tables 2 and 3, respectively. In these tables, $R$-square stands for coefficient of determination and RMSE $\mathrm{A}_{\mathbf{\alpha}}$ (standard error). The estimation of parameters for Clough-Penzien spectrum is better because its RMSE values are smaller and $R$-square values are larger and $>0.5$.

The stationary PSDs at the supports calculated by Eq. (9) using parametric Kanai-Tajimi FRF and Clough-Penzien FRF are shown in Figs. 4(a and b), respectively. The stochastic processes corresponding to these PSDs are used as orthogonal processes at different supports for wavelet-based modeling of spatially varying ground motions employing Eq. (22) and for wavelet-based evolutionary response analyses of the frame employing Eq. (38). The variation of frequency-dependent time-lags between the left support and other supports from site-response effects calculated by using Eq. (8) are shown in Figs. 5(a and b). The time-lags computed by using KanaiTajimi parametric FRF vary by a moderate amount around the

Table 2. Estimated Parameters for Kanai-Tajimi Frequency Response Function

\begin{tabular}{lcccc}
\hline Soil column & $\omega_{s}(\mathrm{rad} / \mathrm{s})$ & $\zeta_{s}(\%)$ & $R$-square & RMSE \\
\hline 1 & 3.218 & 0.334 & 0.525 & 1.966 \\
2 & 5.113 & 0.370 & 0.601 & 1.885 \\
3 & 3.202 & 0.320 & 0.601 & 1.661 \\
4 & 4.765 & 0.362 & 0.597 & 1.790 \\
\hline
\end{tabular}

Note: $R$-square $=$ coefficient of determination for RMSE; $\omega_{s}=$ soil characteristic frequency for Kanai-Tajimi; $\zeta_{s}=$ damping ratio for KanaiTajimi.

12 Table 3. Estimated Parameters for Clough-Penzien FRF

\begin{tabular}{lcccccc}
\hline Soil column & $\omega_{s}(\mathrm{rad} / \mathrm{s})$ & $\zeta_{s}(\%)$ & $\omega_{f}(\mathrm{rad} / \mathrm{s})$ & $\zeta_{f}(\%)$ & $R$-square & RMSE \\
\hline 1 & 3.422 & 0.350 & 0.390 & 0.220 & 0.632 & 1.408 \\
2 & 5.370 & 0.375 & 0.588 & 0.254 & 0.657 & 1.311 \\
3 & 3.278 & 0.323 & 0.344 & 0.140 & 0.601 & 1.532 \\
4 & 4.997 & 0.389 & 0.517 & 0.211 & 0.633 & 1.408 \\
\hline
\end{tabular}

Note: $R$-square $=$ coefficient of determination for RMSE; $\zeta_{f}=$ damping frequency for Clough-Penzien; $\zeta_{s}=$ damping ratio for Kanai-Tajimi; $\omega_{f}$ $=$ soil characteristic frequency for Clough-Penzien; $\omega_{s}=$ soil characteristic frequency for Kanai-Tajimi. frequency of $4 \mathrm{rad} / \mathrm{s}$ whereas the time-lags computed by using Clough-Penzien parametric FRF vary dramatically around a lower frequency of $1 \mathrm{rad} / \mathrm{s}$. This fluctuation in the time-lags in the CloughPenzien FRF case around a lower frequency may have resulted from the additional lower frequency filter [Eq. (7)], which in some cases may be a more realistic representation. The Clough-Penzien FRF is therefore used in the computation for structural responses in the following example even though the Kanai-Tajimi FRF could have been used in the computation with equal ease. For Clough-Penzien FRF, the time-lag peaks of ground motions at support pairs 1 and 2, 1 and 3 , and 1 and 4 are $\sim 2.1,3.5$, and 2.3 s, respectively. Each peak occurs approximately around the average $\omega_{f}$ (see Table 3 ) of the soil columns below the two corresponding supports.

The influence of site-response effects on the amplitude and frequency nonstationarity of the frame-relative displacement responses has been examined. Figs. 6( $a$ and $b$ ) show the RMS values of the relative vertical displacement at the midpoints of the left span and the midspan, respectively calculated by using Eq. (41). When the wavepassage effect is considered alone, the RMS values decrease and attenuate faster in time than those when both wave-passage and siteresponse effects are considered. Similar influence of site-response effects on the relative horizontal displacements at the top of the first and the second columns can be observed in Figs. 7(a and b), respectively. The site-response effects are also shown to alter the amplitude nonstationarity of frame displacements. The increase in the response amplitude nonstationarity from the site-response effect can be evaluated from Figs. 6 and 7. The ratios of the increase in average RMS displacement from the site-response effect, to the average RMS displacement from the wave-passage effect, for the nodes Y6, Y14, X3, and X9 are 41.8703, 42.3704, 39.7965, and $39.7949 \%$, respectively.

The EPSDs of the relative vertical displacements at the midpoints of the left span and the midspan using the parametric CloughPenzien FRF and Eq. (38) are shown in Figs. 8 and 9, respectively. Figs. 10( $\mathrm{a}$ and $\mathrm{b}$ ) show the corresponding PSDs at $t=5 \mathrm{~s}$. The effects of site-response on the amplitude nonstationarity and on reducing the rate of decay of the RMS response envelope values in time, as seen in Fig. 6(b), are also observed in Figs. 8(b) and 9(b). Figs. 10( $a$ and $b$ ) show that compared with the case with only the wave-passage effect considered, the frequency content exhibits stronger nonstationary and the peaks of the instantaneous PSDs are higher in the case of combined wave-passage and site-response effects. In addition, the attenuation of the response energy is slower in time. Similar trends are observed in the EPSD of the column
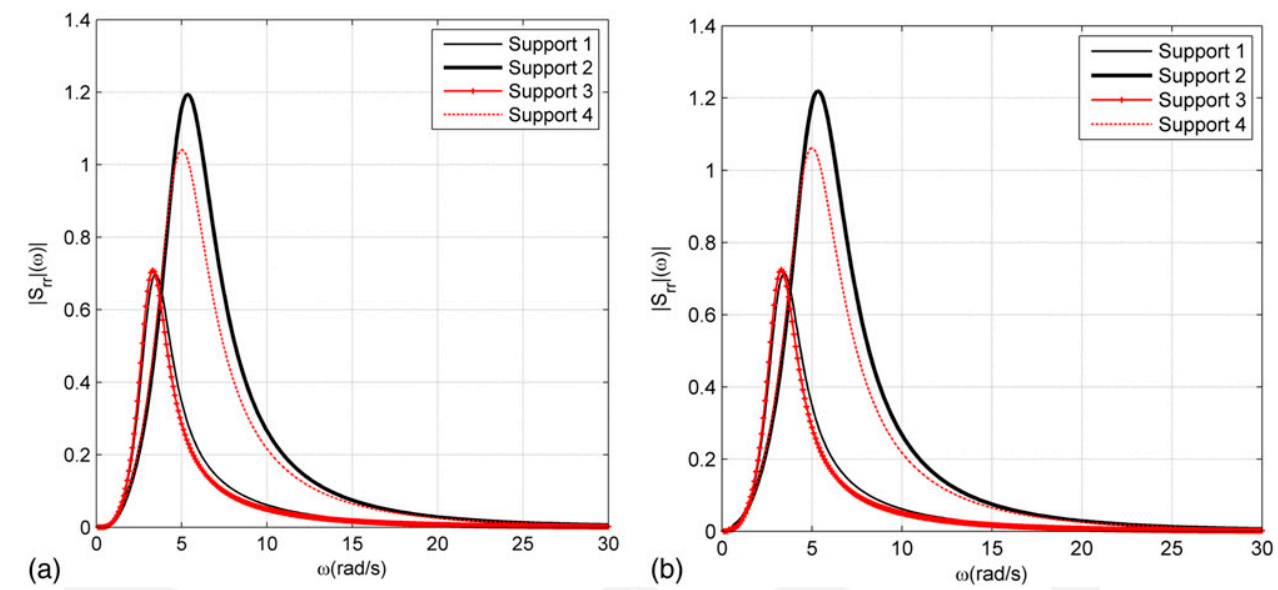

Fig. 4. Orthogonal processes used for different supports: (a) parametric Kanai-Tajimi FRF; (b) parametric Clough-Penzien FRF 

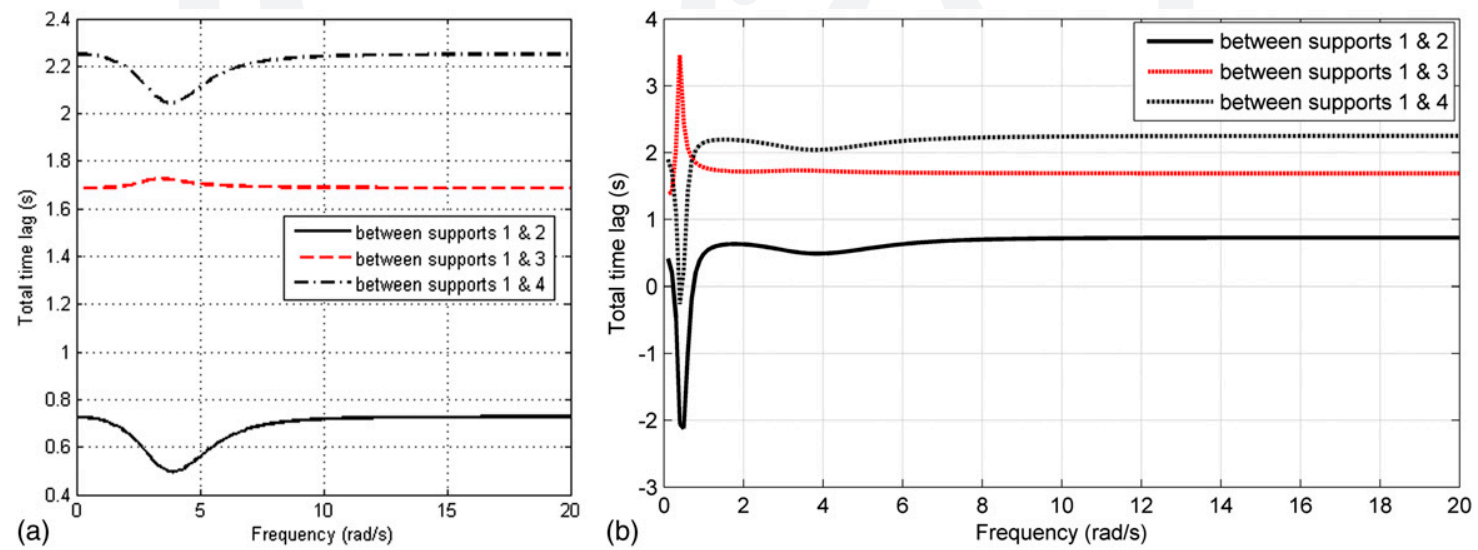

Fig. 5. Variation of frequency-dependent time lags between the left support and other supports from site-response effects: (a) parametric Kanai-Tajimi FRF; (b) parametric Clough-Penzien FRF
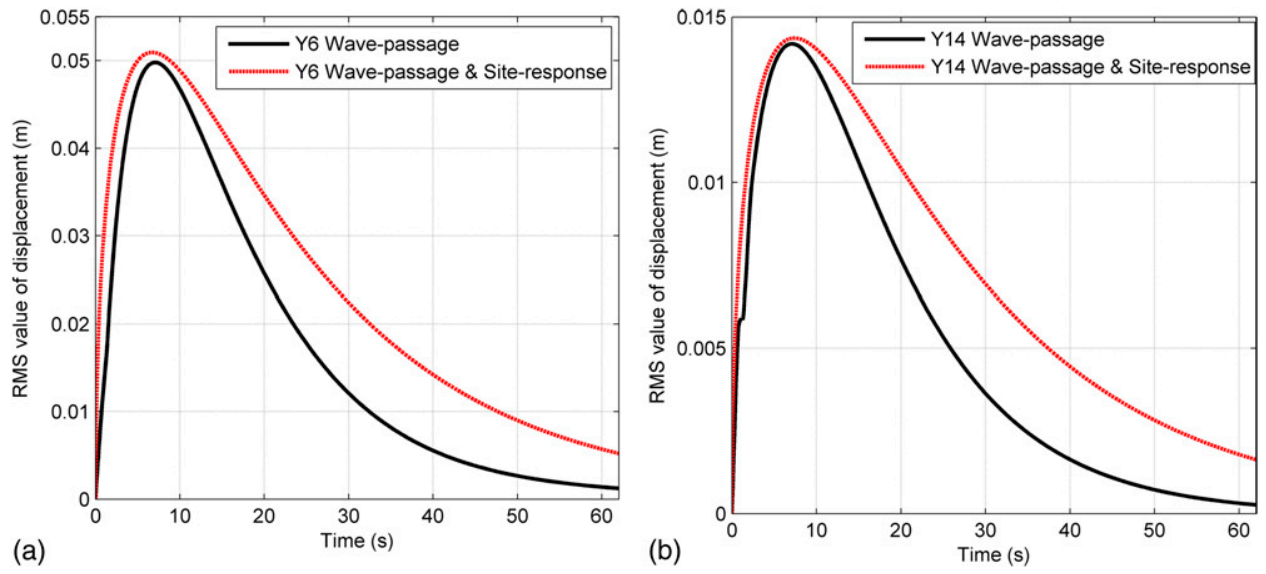

Fig. 6. RMS value of relative vertical displacement: (a) at the midpoint of the left span (Y6); (b) at the midpoint of the midspan (Y14)
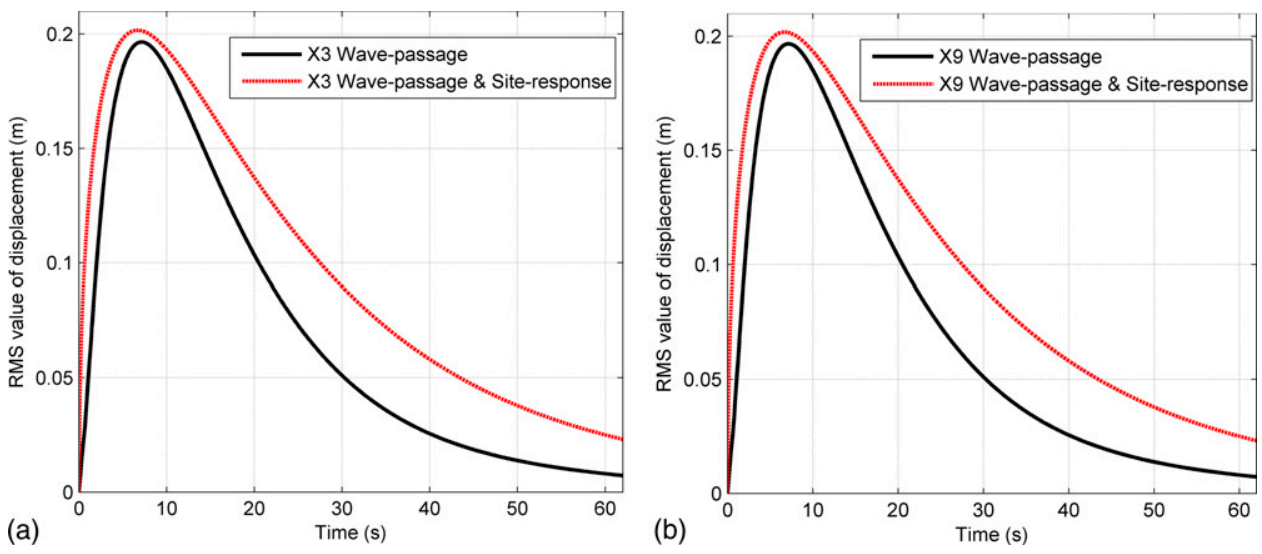

Fig. 7. RMS value of relative horizontal displacement: (a) at the top of the first column (X3); (b) at the top of the second column (X9)

relative horizontal displacements in Fig. 11 and the PSDs at $t=5 \mathrm{~s}$ in Fig. 12.

\section{Conclusions}

A wavelet-based evolutionary response formulation of multispan structures supported on a soil medium and subjected to spatially varying differential support motions including wave-passage and site-response effects has been proposed in this paper. The spatialvariability of support motions is formulated by bedrock parametric coherency models, the time-lags from wave-passage effects, and a proposed alternate way to represent site-response effect by frequency-dependent time-lags. The earthquake energy content is properly characterized by a composite PSDF constituted of parametric 

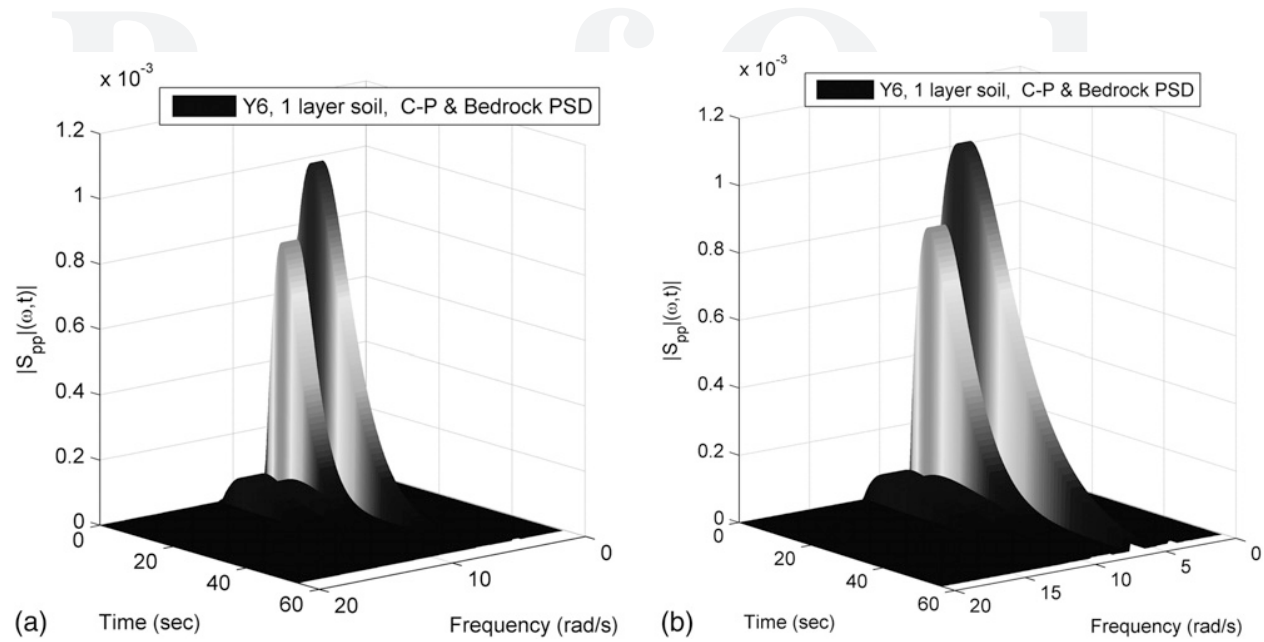

Fig. 8. PSD of relative vertical displacement at midpoint of left span using parametric Clough-Penzien (C-P) frequency response function: (a) wavepassage effect; (b) wave-passage and site-response effects
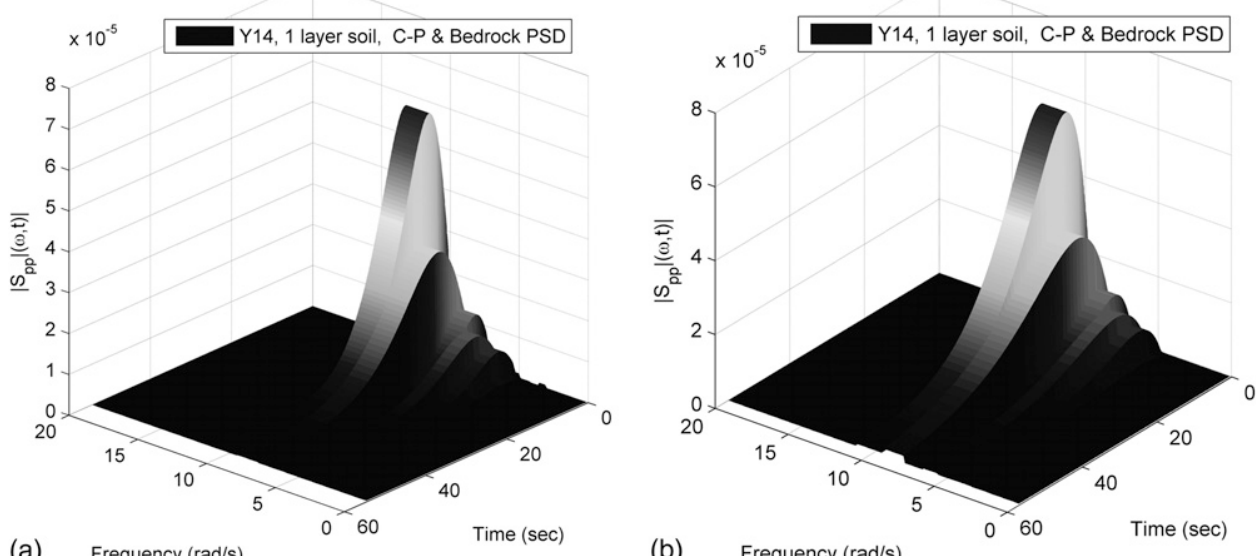

Fig. 9. PSD of relative vertical displacement at midpoint of midspan using parametric Clough-Penzien (C-P) frequency response function: (a) wavepassage effect; (b) wave-passage and site-response effects
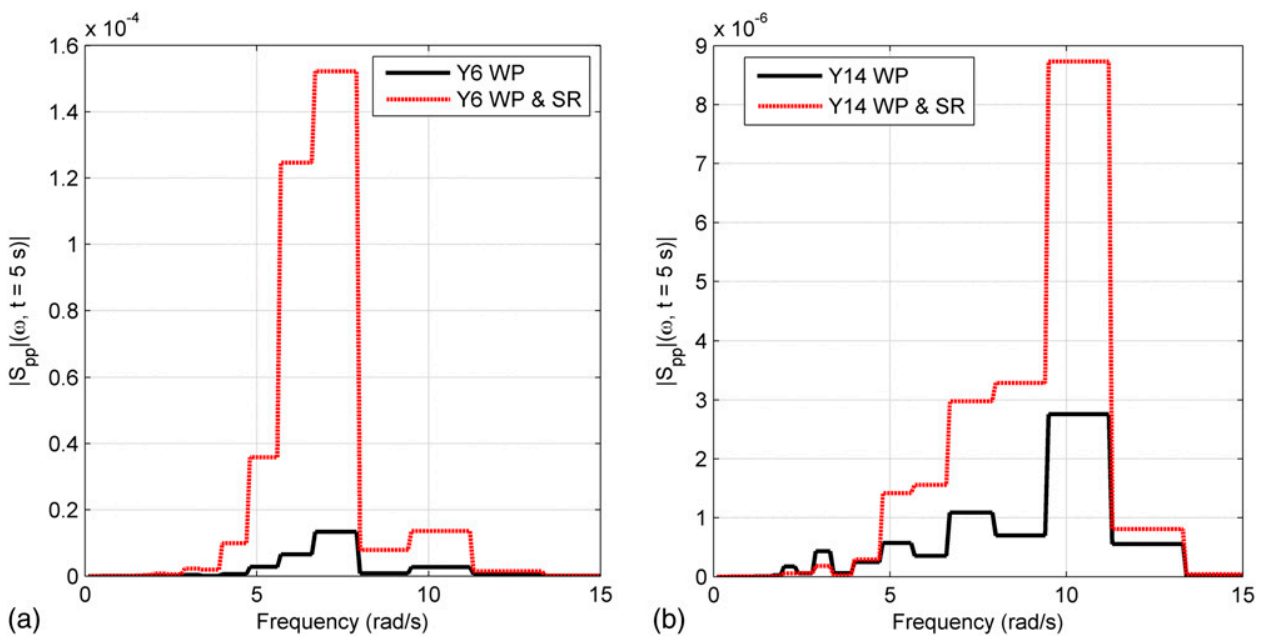

Fig. 10. PSD at $t=5 \mathrm{~s}$ of relative vertical displacements from the wave-passage (WP) effect and wave-passage and site-response (WP and SR) effects: (a) midpoint of left span (Y6); (b) midpoint of midspan (Y14) 

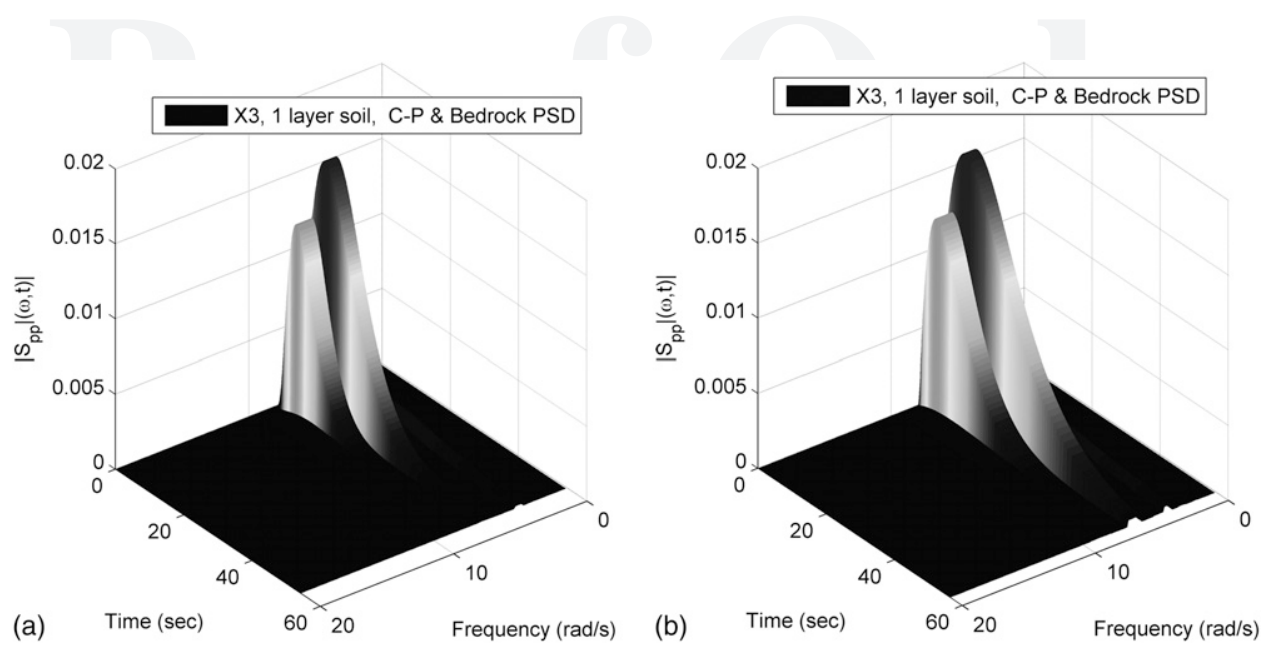

Fig. 11. PSD of relative horizontal displacements at the top of first column using parametric Clough-Penzien (C-P) frequency response function: (a) wave-passage effect; (b) wave-passage and site-response effects
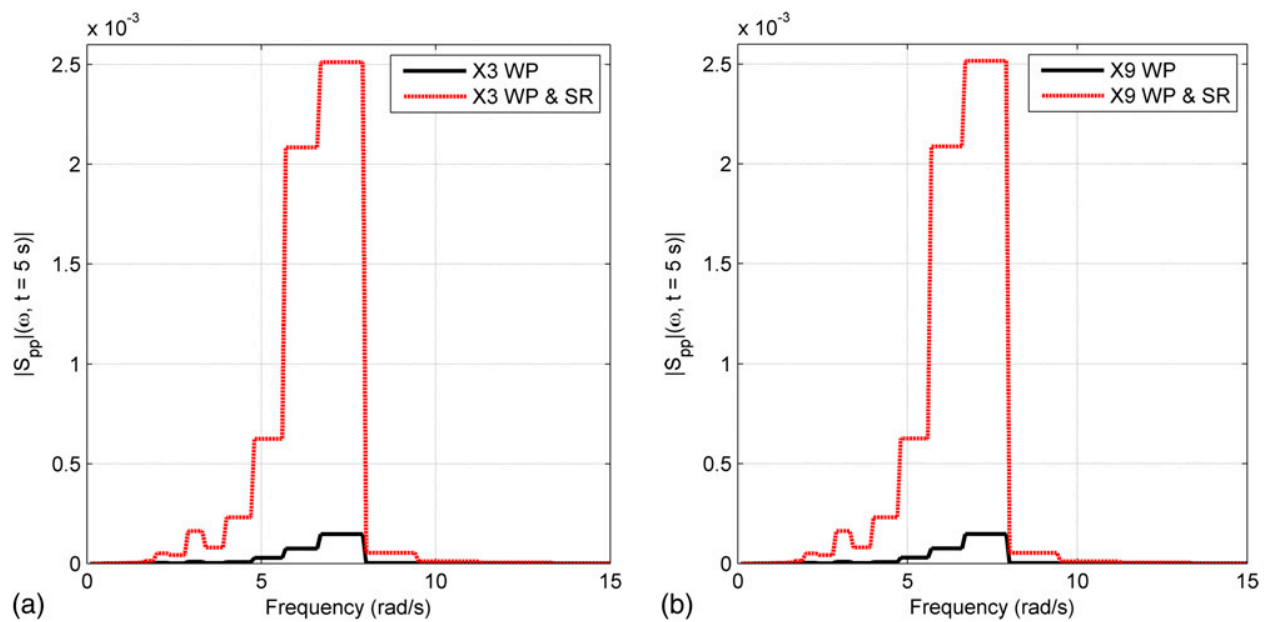

Fig. 12. PSD at $t=5 \mathrm{~s}$ of relative horizontal displacements from the wave-passage (WP) effect and wave-passage and site-response (WP and SR) effects: (a) top of the first column (X3); (b) top of the second column (X9)

surface-to-bedrock FRF and the bedrock power spectrum. The sitecompatible parametric FRFs are proposed to be characterized in this paper by carrying out a FEA of soil media beneath the supports.

In an illustrative case study, a three-span, 2D hangar frame is analyzed using the proposed formulations. The time-lags stemming from the site-response effect, and computed from different FRFs, show different variations in trend. The site-response effect adds frequency nonstationarity to the frame responses and results in an increase of such responses with slower attenuation in time.

This paper proposes a more accurate seismic analysis of longspan multisupport structures because it accounts for the nonstationarities in both amplitude and frequency of excitations and properties and variation of soil media beneath the supports; it offers a more realistic representation of earthquake energy content. The proposed formulations are generally applicable for any waveletbased functions and structures.

\section{Acknowledgments}

This research is partially funded under the EU FP7 Marie Curie IAPP project NOTES (grant No. PIAP-GA- 2008-230663). The authors are grateful for the support. The authors also thank the anonymous reviewers who have given valuable comments.

\section{References}

Basu, B., and Gupta, V. K. (1997). "Non-stationary seismic response of MDOF systems by wavelet modelling of non-stationary processes." Earthquake Eng. Struct. Dynam., 26(12), 1243-1258.

Basu, B., and Gupta, V. K. (1998). "Seismic response of SDOF system by wavelet modeling of non-stationary processes." J. Eng. Mech., 10.1061/ (ASCE)0733-9399(1998)124:10(1142), 1142-1150.

Basu, B., and Gupta, V. K. (2000). "Stochastic seismic response of SDOF systems through wavelets." Eng. Struct., 22(12), 1714-1722.

Boore, D. M. (2003). "Simulation of ground motion using the stochastic method." Pure Appl. Geophys., 160(3-4), 635-676.

Brinkgreve, R. B. J., Broere, D., and Waterman, D. (2008). PLAXIS 2D version 9.0 manual, PLAXIS, Delft, Netherlands.

Chakraborty, A., and Basu, B. (2008). "Nonstationary response analysis of long span bridges under spatially varying differential support motions using continuous wavelet transform.” J. Eng. Mech., 10.1061/(ASCE) 0733-9399(2008)134:2(155), 155-162.

Clough, R. W., and Penzien, J. (2003). Dynamics of structures, McGraw Hill, New York. 
Deodatis, G. (1996). "Non-stationary stochastic vector processes: Seismic ground motion applications.” Probab. Eng. Mech., 11(3), 149-168.

der Kiureghian, A. (1996). "A coherency model for spatially varying ground motions.” Earthquake Eng. Struct. Dynam., 25(1), 99-111.

Dinh, N. V., and Basu, B. (2012). "Zero-pad effects on conditional simulation and application of spatially-varying earthquake motions." Proc., 6th European Workshop on Structural Health Monitoring, 〈www .ewshm2012.com/Portals/98/BB/tu3d3.pdf 7 .

Dumanogluid, A. A., and Soyluk, K. (2003). "A stochastic analysis of long span structures subjected to spatially varying ground motions including the site-response effect." Eng. Struct., 25(10), 1301-1310.

Gurley, K., and Kareem, A. (1999). "Application of wavelet transforms in earthquake, wind, and ocean engineering." Eng. Struct., 21(2), $149-167$.

Hao, H. (1994). "Ground-motion spatial variation effects on circular arch responses." J. Eng. Mech., 10.1061/(ASCE)0733-9399(1994)120:11(2326), 2326-2341.

Hao, H., Olivera, C. S., and Penzien, J. (1989). "Multiple-station ground motion processing and simulation based on SMART-I array data." Nucl. Eng. Des., 111(3), 293-310.

Harichandran, R. S., and Vanmarcke, E. H. (1986). "Stochastic variation of earthquake ground motion in space and time." J. Eng. Mech., 10.1061/ (ASCE)0733-9399(1986)112:2(154), 154-174.

Iyama, J., and Kuwamura, H. (1999). "Applications of wavelets to analysis and simulation of earthquake records." Earthquake Eng. Struct. Dynam., 28(3), 255-272.

Kijewski-Correa, T., and Kareem, A. (2006). "Efficacy of Hilbert and wavelet transforms for time-frequency analysis." J. Eng. Mech., 10.1061/ (ASCE)0733-9399(2006)132:10(1037), 1037-1049.

Konakli, K., and der Kiureghian, A. (2012). "Simulation of spatially varying ground motions including incoherence, wave-passage and site-response effects." Earthquake Eng. Struct. Dynam., 41(3), 495-513.

Kramer, S. L. (1996). Geotechnical earthquake engineering, Prentice Hall, Upper Saddle River, NJ.
Loh, C. H., and Ku, B. D. (1995). "An efficient analysis of structural response for multiple-support seismic excitations." Eng. Struct., 17(1), 15-26.

Luco, J. E., and Wong, H. L. (1986). "Response of a rigid foundation to a spatially random ground motion." Earthquake Eng. Struct. Dynam., 14(6), 891-908.

MATLAB $[$ [Computer software]. Natick, MA, MathWorks.

Priestley, M. B. (1981). Spectral analysis and time series, Academic Press, New York.

Shinozuka, M., and Sato, Y. (1967). "Simulation of nonstationary random processes." J. Eng. Mech. Div., 93(1), 11-40.

Spanos, P. D., and Failla, G. (2004). "Evolutionary spectra estimation using wavelets." J. Eng. Mech., 10.1061/(ASCE)0733-9399(2004)130:8(952), 952-960.

Spanos, P. D., and Kougioumtzoglou, I. A. (2012). "Harmonic wavelets based statistical linearization for response evolutionary power spectrum determination." Probab. Eng. Mech., 27(1), 57-68.

Spanos, P. D., Tezcan, J., and Tratskas, P. (2005). "Stochastic processes evolutionary spectrum estimation via harmonic wavelets." Comput. Methods Appl. Mech. Eng., 194(12-16), 1367-1383.

Tratskas, P., and Spanos, P. D. (2003). "Linear multi-degree-of-freedom system stochastic response by using the harmonic wavelet transform." J. Appl. Mech. 70(5), 724-731.

Trifunac, M. D., and Brady, A. G. (1975). "A study on the duration of strong earthquake ground motion.” Bull. Seismol. Soc. Am., 65(3), 581-626.

Zerva, A. (2009). Spatial variation of seismic ground motions: Modeling and engineering applications, Taylor \& Francis, Boca Raton, FL.

Zerva, A., and Zervas, V. (2002). "Spatial variation of seismic ground motions: An overview." Appl. Mech. Rev., 55(3), 271-297.

Zhang, Y. H., Li, Q. S., Lin, J. H., and Williams, F. W. (2009). "Random vibration analysis of long-span structures subjected to spatially varying ground motions." Soil. Dyn. Earthquake Eng., 29(4), 620-629.

Zhou, Z., and Adeli, H. (2003). "Time-frequency signal analysis of earthquake records using Mexican hat wavelets." Comput. Aided Civ. Infrastruct. Eng., 18(5), 379-389. 


\section{AUTHOR QUERIES}

\section{AUTHOR PLEASE ANSWER ALL QUERIES}

Q: 1_Please check that ASCE Membership Grades (Member ASCE, Fellow ASCE, etc.) are provided for all authors who are members.

Q: 2_Please give a specific reference for "Kanai-Tajimi".

Q: 3_Please give a specific reference for "Clough-Penzien".

Q: 4_The numbered sections and subsections were styled to journal requirements in this paragraph and the one following. Please read the changes carefully and the rewording done to make the paragraph address the sections and subsections in a consistent manner. Kindly check and confirm or amend these changes.

Q: 5_AU: What does "dyn" stand for? Please convert to SI units.

Q: 6_Please give a reference for "Duhamel's integral".

Q: 7_Please spell out "EPSD" here, at first mention.

Q: 8_Please give a date of access for "Dinh and Basu 2012."

Q: 9_Please provide version number for MATLAB.

Q: 10_For Table 1, please check and confirm or amend the defined terms. Please also give a definition for " $E$ " and " $v$ ".

Q: 11_For Table 2, please check and confirm or amend the defined terms.

Q: 12_For Table 3, please check and confirm or amend the defined terms. 